\title{
Die Versorgung wird teurer - und bleibt bezahlbar
}

\section{Jürg Schlup \\ Dr. med., Präsident der FMH}

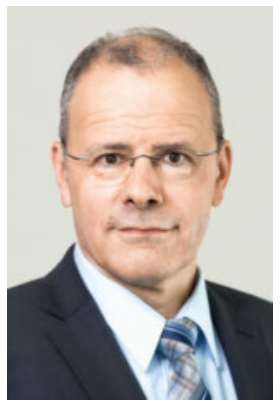

Die Literatur findet sich

unter www.saez.ch

$\rightarrow$ Aktuelle Ausgabe oder

$\rightarrow$ Archiv $\rightarrow 2018 \rightarrow 26 / 27$.
Dass wir Ärzte und keine Ökonomen sind, wird uns zum Nachteil, wenn die Patientenversorgung vor allem unter Kostengesichtspunkten diskutiert wird. So ist es für uns schwierig, ökonomisch unsinnige Behauptungen der Politik entlarven zu können. Auch die aktuell populärste Forderung klingt vordergründig plausibel: Die Gesundheitskosten dürften nicht stärker steigen als die Einkommen, weil sie sonst unbezahlbar würden. Um dies zu verhindern, müsse man einen Kostendeckel einführen. Doch lässt sich diese Forderung überhaupt erfüllen, ohne Behandlungen vorzuenthalten? Ist diese Forderung überhaupt sinnvoll? Erfreulicherweise gibt es Ökonomen, die komplexe Sachverhalte verständlich darstellen können. So erschien 2012 ein Buch des Princeton-Professors William J. Baumol [1], in dem er die bereits 1966 etablierte These zur «Kostenkrankheit» ausführt und erklärt, warum die Gesundheitsausgaben unweigerlich steigen müssen - und warum diese trotzdem bezahlbar bleiben. Diesen sogenannten Baumol-Effekt erläutern zwei Professoren für Wirtschaftswissenschaften in dieser SÄZ auf S. 874 in einem sehr lesenswerten Beitrag [2]. Die Kernidee Baumols war, dass in vielen Arbeitsbereichen - dem sogenannten "progressiven Sektor» - die Produktivität durch Automatisierung ständig wächst, der Output von einer Stunde Arbeit also stetig steigt. In anderen Arbeitsbereichen - dem sogenannten «stagnierenden Sektor» - kann die menschliche Arbeitszeit je-

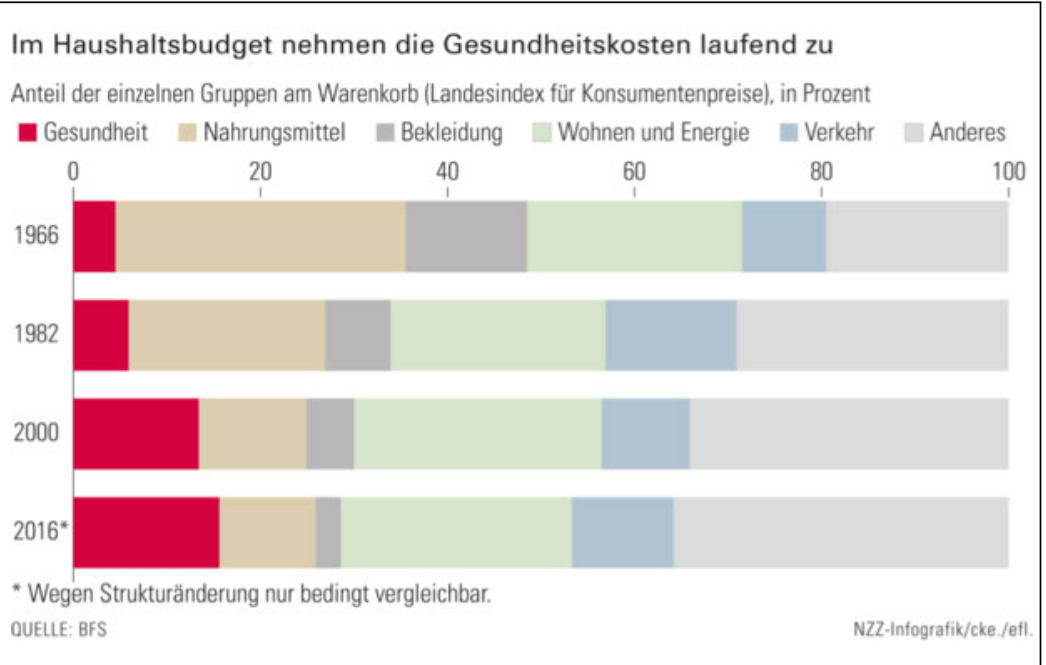

doch kaum reduziert werden. Während also z.B. ein Sack Kartoffeln, eine Jacke oder ein Auto immer weniger Arbeitszeit erfordert, benötigt die Aufführung einer Oper, die Ernährung eines Pflegebedürftigen oder ein Patientengespräch heute noch genauso viel Arbeitszeit wie vor hundert Jahren. Die logische Folge ist, dass die Güter des stagnierenden Sektors relativ zu denen des progressiven Sektors immer teurer werden müssen. Wir werden also relativ immer mehr für Gesundheits- und andere schlecht automatisierbare Leistungen ausgeben müssen - bzw. weniger für die meisten Konsumgüter. Und Letzteres ist die gute Nachricht: Trotz der steigenden Kosten wird Gesundheit immer gesellschaftlich finanzierbar sein, weil ja die steigende Produktivität die Kaufkraft und den Lebensstandard erhöht.

Dies lässt sich an verschiedenen Zahlen nachvollziehen: Während ein Schweizer Haushalt 1966 noch gut $30 \%$ für Nahrung und 13\% für Kleidung ausgab, waren es 2016 nur etwa 10 bzw. 2\% [3]. Für andere Ausgaben verbleibt immer mehr Geld. Ähnlich zeigte auch die Helsana, dass im Zeitraum zwischen 2006 und 2014 die Prämien um 100 Franken, die Ersparnisse hingegen um 715 Franken im Monat stiegen [4].

Heisst das nun, dass es bei den Gesundheitskosten kein Problem gäbe? Nein! Denn auch wenn sich unsere Gesellschaft als Ganzes diese Ausgaben immer besser leisten kann, trifft dies nur im Durchschnitt zu [2, 4]. Während Prämienanstiege alle gleich betreffen, profitieren von den Einkommenszuwächsen nicht alle gleichermassen. Die politisch richtige Schlussfolgerung wäre: Die Lastenverteilung muss verbessert werden! Ein Kostendeckel für die solidarisch finanzierten Gesundheitsausgaben ist die denkbar schlechteste Reaktion: Die folgenden Rationierungen träfen vor allem jene hart, die teurere Dienstleistungen nicht alleine bezahlen können. Der Ökonom Baumol warnte davor, dass Menschen wichtige Leistungen vorenthalten werden, weil sie unerschwinglich scheinen. Darum sei es von zentraler Bedeutung, dass dieser Effekt in der Politik verstanden wird. Denn das Einschränken von Gesundheitsleistungen würde sich zum Nachteil der grossen Mehrheit der Bevölkerung auswirken.

Bildnachweis

NZZ, "Politik muss Debatte über Zweiklassenmedizin aushalten», Ausgabe vom 29.9.2017, S. 13, mit freundlicher Genehmigung der NZZ 